\title{
Beyond Bulk Lifetimes: Insights into Lead Halide Perovskite Films from Time-Resolved Photoluminescence
}

\author{
Florian Staub, ${ }^{1,{ }^{*}}$ Hannes Hempel, ${ }^{2}$ Jan-Christoph Hebig, ${ }^{1}$ Jan Mock, ${ }^{1}$ Ulrich W. Paetzold,,${ }^{1,3,4}$ \\ Uwe Rau, ${ }^{1}$ Thomas Unold, ${ }^{2}$ and Thomas Kirchartz ${ }^{1,5}$ \\ ${ }^{1}$ IEK-5 Photovoltaik, Forschungszentrum Jülich GmbH, 52428 Jülich, Germany \\ ${ }^{2}$ Department Structure and Dynamics of Energy Materials, Helmholtz-Zentrum Berlin für Materialien \\ und Energie GmbH, Hahn-Meitner-Platz 1, 14109 Berlin, Germany \\ ${ }^{3}$ IMEC-Partner in Solliance, Kapeldreef 75, Leuven B-3001, Belgium \\ ${ }^{4}$ Institute of Microstructure Technology, Karlsruhe Institute of Technology, \\ 76344 Eggenstein-Leopoldshafen, Germany \\ ${ }^{5}$ Faculty of Engineering and CENIDE, University of Duisburg-Essen, \\ Carl-Benz-Strasse 199, 47057 Duisburg, Germany
}

(Received 15 June 2016; revised manuscript received 23 August 2016; published 26 October 2016)

\begin{abstract}
Careful interpretation of time-resolved photoluminescence (TRPL) measurements can substantially improve our understanding of the complex nature of charge-carrier processes in metal-halide perovskites, including, for instance, charge separation, trapping, and surface and bulk recombination. In this work, we demonstrate that TRPL measurements combined with powerful analytical models and additional supporting experiments can reveal insights into the charge-carrier dynamics that go beyond the determination of minority-charge-carrier lifetimes. While taking into account doping and photon recycling in the absorber layer, we investigate surface and bulk recombination (trap-assisted, radiative, and Auger) by means of the shape of photoluminescence transients. The observed long effective lifetime indicates high material purity and good passivation of perovskite surfaces with exceptionally low surface recombination velocities on the order of about $10 \mathrm{~cm} / \mathrm{s}$. Finally, we show how to predict the potential open-circuit voltage for a device with ideal contacts based on the transient and steady-state photoluminescence data from a perovskite absorber film and including the effect of photon recycling.
\end{abstract}

DOI: 10.1103/PhysRevApplied.6.044017

\section{INTRODUCTION}

In addition to their high-power-conversion efficiencies in solar cells [1-3], a high-internal-luminescence quantum yield of approximately 30\% [4-6] has been reported for organic-inorganic lead halide perovskites, which suggests remarkably long lifetimes and low defect densities. Their efficient luminescence makes them also promising candidates for light-emitting diodes [5,7] and lasers [8,9]. Because the luminescence of perovskite materials is relatively easy to detect, for instance, with $\mathrm{Si}$ cameras, time-resolved photoluminescence (TRPL) is a suitable and common tool to study charge-carrier dynamics and charge-carrier lifetimes in these materials [10]. Performing TRPL, charge-carrier lifetimes in the range of $10 \mathrm{~ns}$ to over $1 \mu \mathrm{s}$ [11-14] have been reported indicating the formation of perovskite layers with distinctly different defect concentrations.

After excitation with pulsed laser light, photogenerated species undergo various complex mechanisms, like drift, diffusion, trapping, and surface recombination, as well as radiative and nonradiative bulk recombination. Because

*Corresponding author. f.staub@fz-juelich.de these processes often occur at similar times scales subsequent to the excitation pulse, unraveling TRPL data in an accurate way can become challenging $[15,16]$ and photoluminescence (PL) decay curves can easily be misinterpreted. This dilemma results in the availability of diverse rate models $[12,17,18]$ and exponential functions [11,14], which have been used to describe TRPL curves of perovskite materials. With some notable exceptions $[19,20]$, previous reports on transient spectroscopy performed on perovskites usually address only bulk recombination and often leave out surface recombination $[11-13,18]$. We show that these previously derived "bulk" lifetimes might be significantly reduced due to a potentially more prominent charge-carrier recombination at the film surfaces compared to the bulk. Additionally, the effect of photon recycling on obtained lifetimes was not yet considered for the analysis of PL transients. Therefore, radiative recombination constants or lifetimes determined previously from decay dynamics represent the combined effects of recombination (reducing the charge-carrier density) and photon recycling (increasing the charge-carrier density).

The present paper is mainly divided into two parts: First, we present and discuss optical pump terahertz probe (OPTP) measurements, which provide us with chargecarrier mobilities. These mobility values are needed for 
the second part, where we elucidate the impact of chargecarrier recombination on TRPL measurements of a $\mathrm{CH}_{3} \mathrm{NH}_{3} \mathrm{PbI}_{3-x} \mathrm{Cl}_{x}$ perovskite film and present an experimentally verified theoretical TRPL model. Because we consider doping in the absorber layer, we are able to accurately discriminate between high- and low-level injection conditions and, thus, determine reliable recombination parameters of the involved recombination processes. Because reabsorption of photons generated by radiative recombination may lengthen the radiative lifetime significantly [21], we apply corrections to the lifetimes externally obtained by TRPL in order to take into account the impact of photon recycling. Moreover, we show that PL signals with lifetimes of several hundreds of nanoseconds imply a good passivation of perovskite surfaces. Furthermore, with the radiative recombination coefficient in combination with very low absorption coefficients below the band gap deduced from the spectral PL via the generalized Planck emission law, we are able to determine the intrinsic chargecarrier concentration by applying the van RoosbroeckShockley relation. Additional fundamental semiconductor parameters like the effective mass and the effective density of states follow from the intrinsic charge-carrier concentration. Finally, the potential open-circuit voltage is predicted for a perovskite thin film on glass by means of findings from TRPL measurements and by including and quantifying the effect of photon recycling.

\section{EXPERIMENTAL METHODS}

The investigated samples (methylammonium lead iodide perovskite layers on glass substrates) are fabricated by the following procedure: Lead chloride $\left(\mathrm{PbCl}_{2}\right)$ and methylammonium iodide (MAI) are dissolved in dimethylformamide with a molar ratio of MAI: $\mathrm{PbCl}_{2}=3: 1$ (35 wt \%). The solution is spin coated in nitrogen atmosphere on $12 \times 12 \mathrm{~mm}$ Corning glass substrates at $3000 \mathrm{rpm}$ for $35 \mathrm{~s}$ with a ramp time of $10 \mathrm{~s}$. Because a remarkable lifetime enhancement is attributed to the exposure of the film to moisture as well as oxygen [22,23], the samples are annealed in ambient air (36\% relative humidity) on a hot plate at $90^{\circ} \mathrm{C}$ for $2 \mathrm{~h} 20$ min resulting in a $(311 \pm 10)$-nmthick perovskite film. The samples for the individual experiments are fabricated in the same manner to achieve best comparability.

We perform OPTP spectroscopy to characterize the charge-carrier mobilities and ultrafast transients of the perovskite thin film, as reported elsewhere [24]. A 403-nm pump pulse of approximately $70 \mathrm{fs}$ FWHM is used to excite a carrier concentration of $4.8 \times 10^{16} \mathrm{~cm}^{-3}$ in the sample. The conductivity decay is monitored by the transmission of a (0.3-3)-THz probe pulse, which is sensitive to the sample conductivity. Furthermore, the terahertz transmission is modeled by a transfer-matrix method, which yields the sum of electron and hole mobility as a complex spectrum in the terahertz range.
TRPL measurements are performed on samples under inert nitrogen atmosphere. The perovskite film is illuminated with 496-nm light pulses (approximately $1 \mathrm{~ns}$ FWHM) originating from a dye laser operated at $20 \mathrm{~Hz}$. Excitation fluences are varied in the range between $82 \mathrm{~nJ} / \mathrm{cm}^{2}$ and $10 \mu \mathrm{J} / \mathrm{cm}^{2}$ by the use of neutral density filters. PL is spectrally resolved by a SPEX $270 \mathrm{M}$ monochromator from Horiba Jobin Yvon and detected by a gated ICCD camera (iStar DH720 from Andor Solis). The width of the gate pulse, which keeps the multichannel gate open for the PL-related signal to impinge on the Si detector, is chosen between 2 or $5 \mathrm{~ns}$ depending on the signal strength and, thus, on the applied excitation fluence. The temporal resolution of the gate pulse delay is indicated as 25 ps. Because no spectral changes are observed during timeresolved measurements, the recorded spectra are integrated over photon energy to gain the relative photon count at each delay time (time after excitation pulse). Subsequently, the PL signal is normalized to its maximum value and plotted as a function of delay time.

To perform thermoelectric power measurements, coplanar gold contact stripes are thermally deposited onto a Corning glass substrate, and the perovskite layer is formed on top. Heating with an applied temperature gradient of $30 \mathrm{~K}$ results in an actual temperature gradient of approximately $10 \mathrm{~K}$ across the coplanar contact distance of $4 \mathrm{~mm}$. A positive Seebeck coefficient (thermoelectric power) of $S=1042 \mu \mathrm{V} / \mathrm{K}$ is determined in nitrogen atmosphere for an average sample temperature of $335 \mathrm{~K}$. The positive sign of $S$ identifies holes to be the dominant carriers for charge transport. While assuming charge carriers to scatter dominantly at ionized defects, the Seebeck coefficient for a nondegenerated semiconductor is $[25,26]$

$$
S(T)=-\frac{k_{B}}{q}\left(\frac{4 F_{3}\left(\mu^{*}(T)\right)}{3 F_{2}\left(\mu^{*}(T)\right)}-\mu^{*}(T)\right),
$$

where $k_{B}$ denotes the Boltzmann constant, $q$ is the elementary charge, $F_{j}(x)$ is the Fermi-Dirac integral, and $\mu^{*}(T)$ is the reduced Fermi energy at temperature $T$. After solving Eq. (1) for $\mu^{*}(T)$, we are able to calculate a hole concentration $p(T)$ by using

$$
p(T)=N_{v}(T) F_{1 / 2}\left(\mu^{*}(T)\right) .
$$

Here, the density of states in the valence band $N_{v}$ is assumed as $N_{v}(T) \approx 1.5 \times 10^{15} \mathrm{~T}^{3 / 2} \mathrm{~cm}^{-3} \mathrm{~K}^{-3 / 2}$ based on the values listed in Table II.

Photothermal deflection spectroscopy (PDS) is used as a highly sensitive technique to obtain absorption coefficients of a perovskite film on glass substrate in a dynamic range of several orders of magnitude. A detailed description of this method can be found elsewhere [27,28]. The sample is measured in a cuvette filled with a FC-75 solution, which is 
chemically inert towards organic compounds and exhibits a temperature-dependent refractive index. A halogen lamp $(100 \mathrm{~W})$ in combination with a spectrometer $(270 \mathrm{M}$ from Horiba Jobin Yvon) illuminates the sample with monochromatic light in transverse mode. Any absorbed photon energy by the sample is eventually converted into heat, which is released to the surrounding media. A probe beam from a diode laser $(650 \mathrm{~nm})$ adjusted in parallel to the sample surface is deflected due to the gradient in the refractive index of the chemical. Finally, the deflection angle monitored by a silicon four-quadrant diode is directly proportional to the power of the absorbed light. In order to gain absolute values of the absorption coefficient, the transmittance is additionally measured, and the refractive index as well as the thickness of the absorbing layer have to be known.

\section{RESULTS AND DISCUSSION}

\section{A. Charge-carrier mobilities derived from optical pump terahertz probe spectroscopy}

We perform OPTP spectroscopy measurements on a $\mathrm{CH}_{3} \mathrm{NH}_{3} \mathrm{PbI}_{3-x} \mathrm{Cl}_{x}$ perovskite layer deposited on a glass substrate to determine charge-carrier mobilities and to help interpret transient photoluminescence data. A detailed description of the OPTP technique can be found elsewhere [24]. Here, an approximately 100-fs pump pulse with a wavelength of $403 \mathrm{~nm}$ generates an initial electron and hole concentration of $\Delta n=\Delta p=4.8 \times 10^{16} \mathrm{~cm}^{-3}$. The measured pump-induced change in terahertz probe pulse transmission $\Delta E / E$ is directly proportional to the conductivity change $\Delta \sigma$ according to the equation

$$
\frac{\Delta E(t)}{E} \propto \Delta \sigma(t)=q\left[\mu_{n} \Delta n(t)+\mu_{p} \Delta p(t)\right] .
$$

Here, $q$ is the elementary charge, while $\mu_{n}$ and $\mu_{p}$ refer to the electron and hole mobility, respectively. For the exact relation between $\Delta E / E$ and $\Delta \sigma$, we model the transmitted terahertz radiation based on a transfer-matrix method and, thus, obtain the sum of electron and hole mobility.
Figure 1(a) indicates that the measured pump-induced conductivity (blue line) stays constant within the first $2 \mathrm{~ns}$ after photoexcitation. This behavior is in contrast to OPTP transients of many other evolving thin-film photovoltaic materials like $\mathrm{Cu}_{2} \mathrm{SnS}_{3}$ [29], $\mathrm{Cu}_{2} \mathrm{ZnSn}(\mathrm{S}, \mathrm{Se})_{4}$ [30], and microcrystalline silicon [31]. Typically, a fast decay on the pico- to nanosecond time scale is observed, which is caused by charge-carrier recombination. Here, no conductivity decay can be resolved within the time frame of the OPTP measurement ( $2 \mathrm{~ns}$ ), indicating constant charge-carrier concentrations due to low recombination rates. This finding is consistent with the work of Milot et al. [32] for low applied excitation fluences. Figure 1(b) shows the complex mobility, which accounts for the sum of electron and hole contributions measured by OPTP at a delay time of $1 \mathrm{~ns}$. The real part of the mobility stays constant (approximately $40 \mathrm{~cm}^{2} \mathrm{~V}^{-1} \mathrm{~s}^{-1}$ ) over the entire frequency range and, thus, can be extrapolated to the same dc value. The observed dip in the imaginary mobility at about $1 \mathrm{THz}$ is previously assigned to the coupling of terahertz radiation to a phonon mode [33]. Because the effective masses for electrons and holes in organic-inorganic lead halide perovskites are predicted to be similar [34], we assume equal electron and hole mobilities of approximately $20 \mathrm{~cm}^{2} \mathrm{~V}^{-1} \mathrm{~s}^{-1}$. The high-charge-carrier mobility values derived from OPTP measurements are similar to previously reported values $[35,36]$.

\section{B. Time-resolved photoluminescence \\ 1. Modeling photoluminescence decays considering doping}

Normalized PL transients of a $\mathrm{CH}_{3} \mathrm{NH}_{3} \mathrm{PbI}_{3-\mathrm{x}} \mathrm{Cl}_{\mathrm{x}}$ perovskite film on a glass substrate are shown in Fig. 2 for different excitation fluences ranging over more than 2 orders of magnitude.

Upon photoexcitation, the only species generated in $\mathrm{CH}_{3} \mathrm{NH}_{3} \mathrm{PbI}_{3}$ perovskites will be free charge carriers with concentration $\Delta n$ because the determined values for exciton binding energies of only approximately $2-16 \mathrm{meV}$ [37-40] are smaller than the available thermal energy at room temperature.
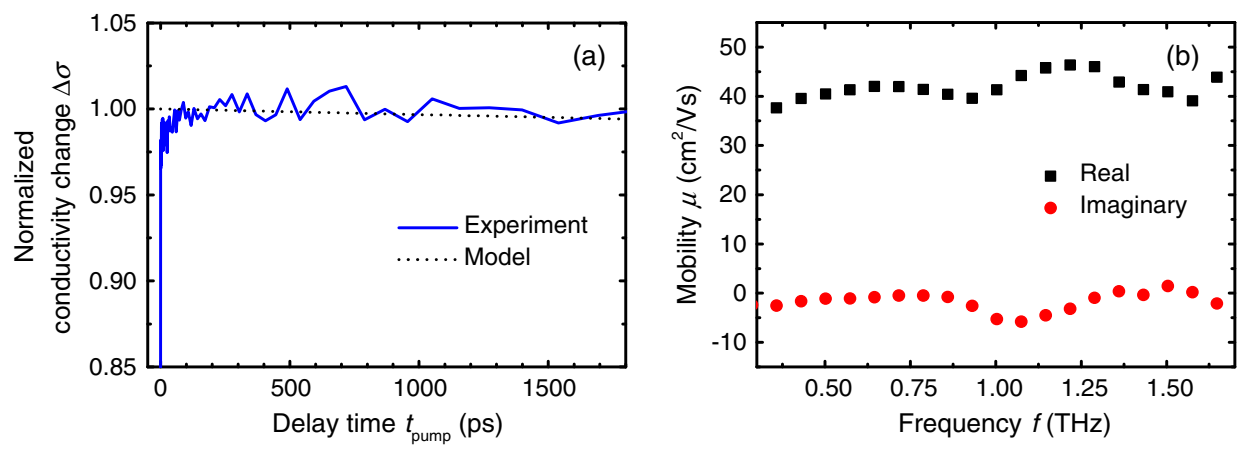

FIG. 1. Optical pump terahertz probe spectroscopy measurements of a $\mathrm{CH}_{3} \mathrm{NH}_{3} \mathrm{PbI}_{3-x} \mathrm{Cl}_{x}$ perovskite film. (a) Measured normalized conductivity change $\Delta \sigma$ of the sample over delay time $t_{\text {pump }}$ (blue line). The black dotted line represents the modeled conductivity change. (b) Real and imaginary parts of the derived complex mobility $\mu$ for different probe frequencies $f$ at $1 \mathrm{~ns}$ after photoexcitation. The real part accounts for the sum of electron and hole mobility. 


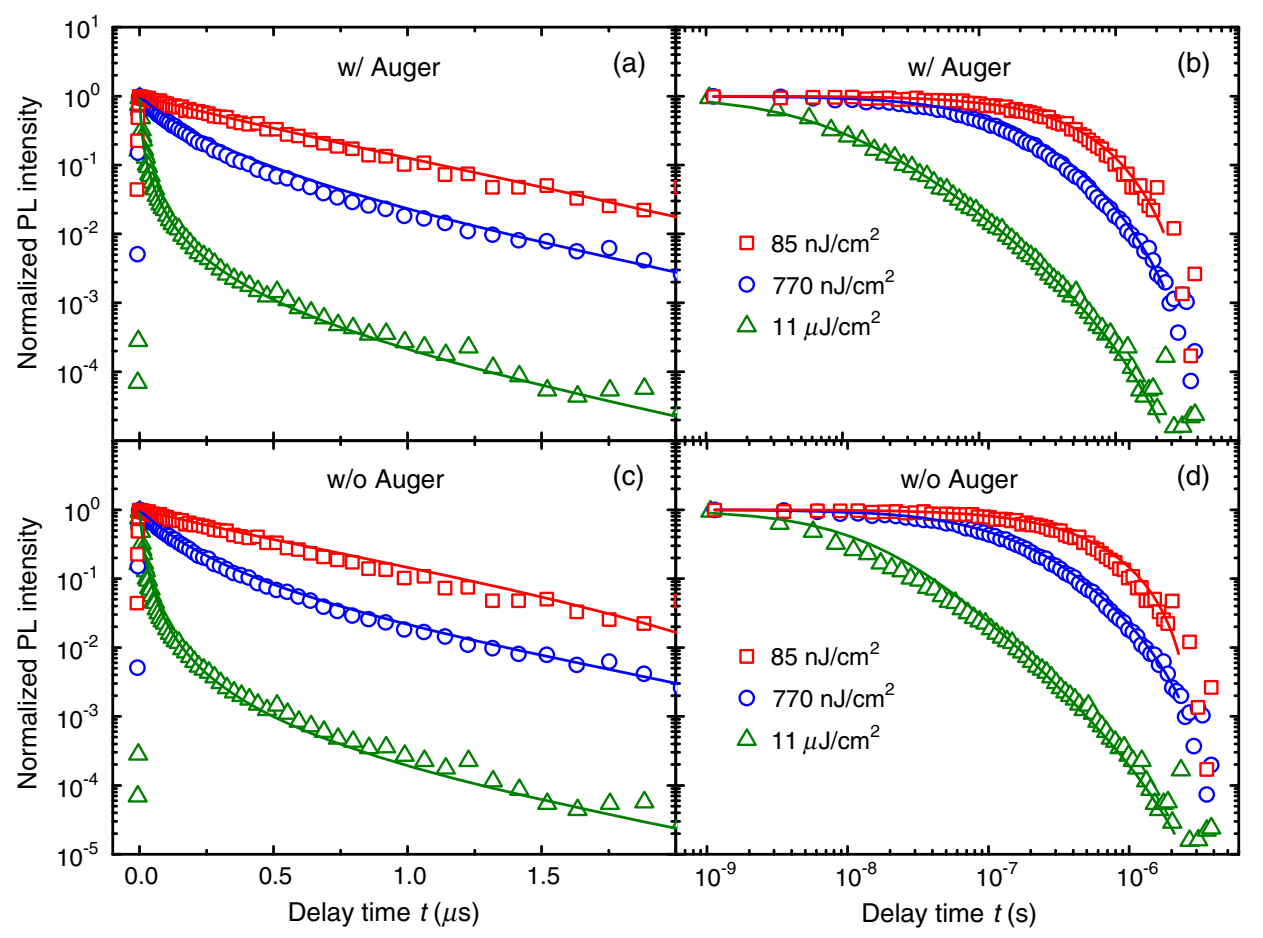

FIG. 2. Normalized photoluminescence decays from measurements (open symbols) of a $\mathrm{CH}_{3} \mathrm{NH}_{3} \mathrm{PbI}_{3-x} \mathrm{Cl}_{x}$ perovskite film for different excitation fluences: (a),(c) on a semilogarithmic scale, while (b),(d) on a double-logarithmic scale. Solid lines represent global fits including radiative and trap-assisted recombination for the bottom row (w/o Auger) and Auger recombination for the top row (w/ Auger).

As a first approach, we consider only charge-carrier recombination in the bulk and leave out the effect of surface recombination, which we discuss separately in Sec. III B 5.

The excess-charge-carrier density $\Delta n(t)$ at time $t$ after the excitation pulse is described by the continuity equation

$$
\begin{aligned}
\frac{\partial}{\partial t} \Delta n= & -R+G_{\text {int }}=-k\left(n p-n_{i}^{2}\right)-\frac{n p-n_{i}^{2}}{\tau_{p} n+\tau_{n} p} \\
& -C_{n} n\left(n p-n_{i}^{2}\right)-C_{p} p\left(n p-n_{i}^{2}\right)+G_{\text {int }},
\end{aligned}
$$

where $R$ is the recombination rate, $G_{\text {int }}$ is the generation rate due to radiative recombination in the absorber layer, $k$ is the radiative recombination coefficient, $\tau_{n}$ and $\tau_{p}$ are the Shockley-Read-Hall (SRH) lifetimes for electrons and holes, and $C_{n}$ and $C_{p}$ are the Auger coefficients for electrons and holes. The electron and hole concentrations in Eq. (4) are expressed as $n=n_{0}+\Delta n$ and $p=p_{0}+\Delta n$, respectively, with the corresponding equilibrium concentrations $n_{0}$ and $p_{0}$. The intrinsic charge-carrier concentration is denoted with index $i$ and is neglected in the following. The first term on the right-hand side of Eq. (4) corresponds to radiative band-to-band recombination of free electrons and free holes with the externally observed radiative recombination coefficient $k$. Photons generated by radiative recombination can be reabsorbed by the perovskite layer with probability $p_{r}$. This process, often referred to as photon recycling (PR), causes a quasi-immediate internal generation of electron-hole pairs $G_{\text {int }}$, and we find an apparent radiative recombination rate:

$$
k n p-G_{\mathrm{int}}=\left(k-p_{r} k\right) n p=k^{*} n p .
$$

According to that, the external radiative recombination coefficient typically obtained from the TRPL measurement is $k^{*}$, and in order to derive the internal radiative recombination coefficient $k$, we use

$$
k=\frac{k^{*}}{\left(1-p_{r}\right)} .
$$

Hence, the apparent, external radiative lifetime appears substantially longer than the internal radiative lifetime, which is a well-known fact from GaAs-based devices [21].

To obtain the temporal excess-charge-carrier concentration $\Delta n(t)$, Eq. (4) has to be solved numerically. TRPL detects radiative recombination and, consequently, is given by

$$
\operatorname{PL}(t)=k^{*}\left\{\left[n_{0}+\Delta n(t)\right]\left[p_{0}+\Delta n(t)\right]-n_{i}^{2}\right\} .
$$

The obtained lifetimes from modeling PL decays will depend on the doping concentration in the sample: the longterm decay is either limited by the minority-charge-carrier lifetime or the product of the doping concentration and radiative recombination parameter [41]. The doping concentration cannot be obtained easily just by fitting the TRPL transients. Good fits can be achieved for doping concentrations up to a maximum limit of about $4 \times 10^{16} \mathrm{~cm}^{-3}$, whereas the values of resulting lifetimes change depending on the doping density assumed. Therefore, the doping densities have to be measured separately. We perform thermoelectric power measurements in nitrogen atmosphere, 
TABLE I. Bulk-recombination parameters obtained from modeling time-resolved photoluminescence decays for a fixed $p$-type doping concentration of $p_{0}=3 \times 10^{15} \mathrm{~cm}^{-3}$ : the external radiative recombination coefficient $k^{*}$ as well as its internal counterpart $k$, which is corrected for photon recycling, the minority- and majority-charge-carrier lifetimes $\tau_{n}$ and $\tau_{p}$ from trap-assisted Shockley-Read-Hall recombination, as well as the Auger recombination coefficient $C=C_{n}+C_{p}$. The commonly stated lifetime $\tau_{e}$, which is obtained from the monoexponential timeresolved photoluminescence regime at long times, is in excellent agreement with the minority-charge-carrier lifetime $\tau_{n}$.

\begin{tabular}{lccccc}
\hline \hline$k^{*}\left(\mathrm{~cm}^{3} \mathrm{~s}^{-1}\right)$ & $k\left(\mathrm{~cm}^{3} \mathrm{~s}^{-1}\right)$ & $\tau_{n}(\mathrm{~ns})$ & $\tau_{p}(\mathrm{~ns})$ & $C\left(\mathrm{~cm}^{6} \mathrm{~s}^{-1}\right)$ & $\tau_{e}(\mathrm{~ns})$ \\
\hline \hline$(4.78 \pm 0.43) \times 10^{-11}$ & $(8.77 \pm 0.79) \times 10^{-10}$ & $511 \pm 80$ & $871 \pm 251$ & $(8.83 \pm 1.57) \times 10^{-29}$ & $508 \pm 29$ \\
\hline \hline
\end{tabular}

which reveal a positive Seebeck coefficient (thermoelectric power) of $S=1042 \mu \mathrm{V} / \mathrm{K}$ at $335 \mathrm{~K}$. From that value, we derive a moderate $p$-type doping with a concentration of $p_{0} \approx 3 \times 10^{15} \mathrm{~cm}^{-3}$ (see Sec. II for more details). This value is in good agreement with previous findings from Hall measurements [35] and electron-beam-induced current profiles [42], suggesting weakly doped for stoichiometric $\mathrm{CH}_{3} \mathrm{NH}_{3} \mathrm{PbI}_{3}$ as well as $\mathrm{CH}_{3} \mathrm{NH}_{3} \mathrm{PbI}_{3-x} \mathrm{Cl}_{x}$ perovskites. The normalized TRPL decays in Figs. 2(a) and 2(b) are globally fitted according to Eqs. (4) and (7) by the method of least squares for the obtained doping concentration of $p_{0}=3 \times 10^{15} \mathrm{~cm}^{-3}$ and for neglecting $n_{0}$ and $n_{i}$. Additionally, the resulting fits by a model without Auger recombination are shown in Figs. 2(c) and 2(d). As can be seen, a better conformity with experimental data for the fits with Auger recombination is observed for the highest applied excitation fluence at short times when Auger processes are affecting the PL decay. The resulting fitting parameters from the model including Auger recombination, which are listed in Table I, are in good agreement with previously reported values $[12,36,43]$. Based on the derived set of recombination parameters from TRPL analysis, we model the normalized conductivity change according to Eq. (3), and, thus, we are able to predict the obtained OPTP trace [black dotted line in Fig. 1(a)].

For our sample, the influence of Auger recombination on the TRPL curves is seen only for high-level injection conditions, when $\Delta n \gg p_{0} \gg n_{0}$ holds. Therefore, we can provide only the sum of the individual Auger coefficients $C=C_{n}+C_{p}$ instead of values for each carrier type. Additionally, please note that the majority-charge-carrier lifetime $\tau_{p}$ has a much higher uncertainty than the minoritycharge-carrier lifetime because $\tau_{p}$ affects the PL transients only slightly during a short time interval (see, also, Fig. 3). Moreover, we show in Fig. 3(a) what range of relative PL intensities and excess-charge-carrier concentrations is covered by our data. The initial photogenerated chargecarrier concentrations $\Delta n(t=0)$ can be calculated from the corresponding applied excitation fluences. Over time, the excess-charge-carrier concentration is reduced due to recombination events. By means of Eq. (4) in combination with the derived recombination parameters, we find for

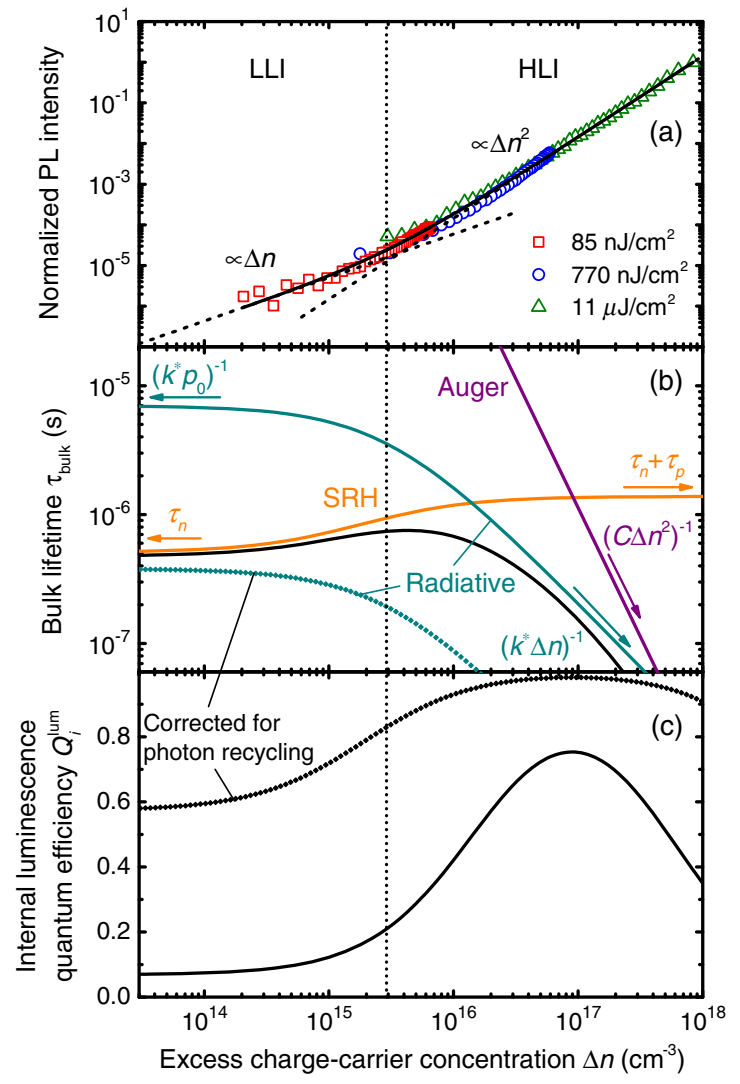

FIG. 3. (a) Normalized photoluminescence intensity as a function of the injected excess-charge-carrier concentration $\Delta n$ derived from solving the continuity equation. Open symbols represent experimental data for the indicated excitation fluences. The solid line shows the best fit from simulation. Furthermore, dashed lines illustrate regions where low level injection (LLI) and high level injection (HLI) conditions hold. Here, the dotted black line displays the excess-charge-carrier concentration under illumination corresponding to an intensity of one sun. (b) Bulk lifetimes of the individual recombination processes: SRH, Auger, and radiative recombination without (solid line) and with corrections due to photon recycling (diamonds). The black curve shows the effective bulk lifetime $\tau_{\text {bulk }}$. (c) Apparent internal luminescence quantum efficiency $Q_{i}^{\text {lum }}$ (solid line) and corrected for photon recycling (diamonds). 
each selected delay time $t>0$ the current excess-chargecarrier concentration $\Delta n(t)$ and attribute this value to the respective measured PL intensity. Additionally, we adjust the obtained PL intensities of each data set (different excitation fluences) relative to the others. The relative PL data depending now on $\Delta n$ are shown in Fig. 3(a) and follow the trend of Eq. (7): for high-level-injection (HLI) conditions $\Delta n \gg p_{0} \gg n_{0}, n_{i}$, the PL intensity scales with $\Delta n^{2}$, whereas in the low-level-injection (LLI) regime $p_{0} \gg \Delta n \gg n_{0}, n_{i}$, the PL signal is rather proportional to $\Delta n$.

\section{Effect of photon recycling on the radiative lifetime}

As stated in Eq. (6), we account for photon recycling by dividing the external radiative recombination coefficient $k^{*}$ by $1-p_{r}$ in order to derive a higher internal coefficient $k$. Here, we assume that parasitic absorption can be neglected for an absorber film on glass, and, thus, $1-p_{r}$ matches the outcoupling efficiency $p_{e}$, which can be calculated as the ratio of the outgoing photon flux to the internal radiative recombination in the layer [44]. For a thin film on glass, emitted photons can escape the sample from both sides, and we find $p_{e}$ increases by a factor of 2 compared to Ref. [44],

$$
p_{e}=\frac{\int_{0}^{\infty} A(E) \phi_{\mathrm{BB}}(E) d E}{2 d \int_{0}^{\infty} n_{r}^{2}(E) \alpha(E) \phi_{\mathrm{BB}}(E) d E},
$$

with $A$ being the absorptance of the perovskite film with thickness $d$ and $\alpha$ being the absorption coefficient (see, also, Sec. III B 6). Furthermore, $n_{r}$ denotes the refractive index, and $\phi_{\mathrm{BB}}$ is the spectral black-body radiation expressed as

$$
\phi_{\mathrm{BB}}(E)=\frac{2 E^{2}}{h^{3} c^{2}} \frac{1}{\exp \left[E /\left(k_{B} T\right)\right]-1},
$$

where $h$ is Planck's constant, $c$ is the speed of light in vacuum, $k_{B}$ is the Boltzmann constant, and $T$ is the temperature of the sample. For the examined layer, we derive an outcoupling efficiency of $p_{e} \approx 0.055$ from Eq. (8), and the internal radiative recombination coefficient $k$ is more than 18 -fold higher than the externally obtained $k^{*}$ (see Table I).

\section{Charge-carrier lifetimes of recombination processes}

With the obtained recombination parameters from fitting of the recombination kinetics, we derive an apparent effective bulk lifetime $\tau_{\text {bulk }}$ accounting for all bulkrecombination mechanisms simply by

$$
\tau_{\text {bulk }}=\frac{\Delta n}{R-G_{\text {int }}} .
$$

Additionally, we define lifetimes for each of the individual recombination processes. As depicted in Fig. 3(b), these lifetimes strongly depend on the excess-charge-carrier concentration $\Delta n$ and, therefore, on the illumination intensity. The effective bulk lifetime [Fig. 3(b), black solid line] is mostly influenced by SRH recombination at moderate excess-charge-carrier concentrations $\Delta n \lesssim 10^{16} \mathrm{~cm}^{-3}$. At higher-charge-carrier concentrations, radiative recombination, and, subsequently, Auger recombination are dominant. As stated in Sec. III B 2 and seen in Fig. 3(b), the radiative lifetime corrected for photon recycling becomes significantly shorter (dark cyan diamonds).

In the next step, we derive the excess-charge-carrier concentration $\Delta n$ generated upon steady-state illumination of one-sun intensity. We use the numerical device simulator Advanced Semiconductor Analysis (ASA) [45] in order to calculate the (external) optical generation rate $G_{\text {ext }}$ in the perovskite absorber layer embedded in a hypothetical device stack, while considering interference effects: glass $(1 \mathrm{~mm}) / \mathrm{ITO} \quad(120 \mathrm{~nm}) /$ PEDOT $(25 \mathrm{~nm}) /$ perovskite $(311 \mathrm{~nm}) / \mathrm{PCBM} \quad(60 \mathrm{~nm}) / \mathrm{ZnO}-\mathrm{NP} \quad(60 \mathrm{~nm}) / \mathrm{Al}$ $(150 \mathrm{~nm})$. Optical data of these materials (refractive indices $n_{r}$ and extinction coefficients $k$ ) can be found in the Supplemental Material [41]. Upon steady-state illumination at open-circuit conditions, the obtained generation rate $G_{\text {ext }} \approx 3.9 \times 10^{21} \mathrm{~cm}^{-3} \mathrm{~s}^{-1}$ matches the recombination rate. Solving for $\Delta n$ yields an excess-charge-carrier concentration of $\Delta n \approx 2.9 \times 10^{15} \mathrm{~cm}^{-3}$ at one sun. Figure 3(c) shows the internal luminescence quantum efficiency $Q_{i}^{\text {lum }}$, which is defined by

$$
Q_{i}^{\text {lum }}=\frac{R_{\text {rad }}}{R_{\text {rad }}+R_{\text {nrad }}}
$$

as a function of the excess-charge-carrier concentration $\Delta n$. First, $Q_{i}^{\text {lum }}$ rises in both cases (with and without corrections for photon recycling) with increasing $\Delta n$, before it starts to drop due to the beginning influence of Auger recombination. Allowing for photon recycling causes a distinct boost in the internal luminescence quantum efficiency, and values are approaching unity for excess-charge-carrier concentrations of $\Delta n \approx 10^{17} \mathrm{~cm}^{-3}$. At one-sun illumination, charge carriers radiatively recombine with a corrected high quantum yield of $Q_{i}^{\text {lum }} \approx 0.80$, whereas the internal luminescence quantum efficiency appears to be only $Q_{i}^{\text {lum }} \approx 0.20$ without photon recycling.

\section{Charge-carrier diffusion length}

In the next step, we calculate the diffusion length of minority charge carriers $L_{d}$ by

$$
L_{d}=\sqrt{D \tau_{\text {bulk }}},
$$

where $\tau_{\text {bulk }}$ refers here to the effective bulk lifetime at onesun illumination $\left(\tau_{\text {bulk }} \approx 740 \mathrm{~ns}\right)$. The diffusion constant $D$ 
can be expressed in terms of the derived charge-carrier mobility of $\mu=20 \mathrm{~cm}^{2} / \mathrm{Vs}$ by the Einstein-relation as

$$
D=\frac{\mu k_{B} T}{q},
$$

with $k_{B} T$ being the thermal energy and $q$ the elementary charge. Consequently, we find a diffusion length of $L_{d} \approx 6 \mu \mathrm{m}$, which substantially exceeds typical absorber layer thicknesses.

\section{Surface recombination: Lifetimes and velocities}

So far, we consider only recombination in the bulk of the perovskite absorber layer. However, defects can be located not only in the bulk but also at the surfaces of the absorber layer in the form of, e.g., dangling bonds and uncoordinated atoms. Therefore, we introduce an additional SRH recombination rate accounting solely for recombination at surfaces, which reads for electrons as minority charge carriers [15]:

$$
\tilde{R}_{s}=\frac{\Delta n_{s}\left(p_{0}+\Delta n_{s}\right)}{\frac{\Delta n_{s}}{S_{p}}+\frac{p_{0+} \Delta n_{s}}{S_{n}}} .
$$

When we assume that the defect density in the bulk is sufficiently low, the local excess-charge-carrier concentration $\Delta n_{s}$ at the surfaces will always be lower than in the bulk due to ongoing surface recombination. Because of the low $p$-type doping found in the examined sample, $\Delta n_{s}$ can still be larger than $p_{0}$ for high excitation fluences. However, when we assume equal surface recombination velocities for electrons and holes $S_{n}=S_{p}=S$, we find

$$
\tilde{R}_{s} \approx \xi S \Delta n_{s},
$$

with $\xi$ varying between 0.5 and 1 depending on the prevalent injection level in the sample (HLI, $\xi=0.5$ and LLI, $\xi=1$ ). For simplicity, $\xi=1$ is assumed in the following calculations. Because $\tilde{R}_{s}$ denotes the number of charge carriers recombining per surface area and time, we rather transform Eq. (15) into a bulk-recombination rate $R_{s}$ :

$$
R_{s} \approx \xi \frac{S}{d} \Delta n_{s}=\frac{\Delta n}{\tau_{s}}
$$

Moreover, the excess-charge-carrier concentration $\Delta n_{s}$ at the surfaces of the perovskite thin film is related to the concentration $\Delta n$ in the bulk as a function of $\xi$, the layer thickness $d$, the surface recombination velocities $S$ at both surfaces, and the diffusion constant $D$. As a consequence, a surface lifetime $\tau_{s}$ with respect to $\Delta n$ can be defined and is obtained by solving the continuity equation with respect to the boundary conditions $[46,47]$.
The overall effective lifetime is expressed as being made up of a bulk and a surface contribution,

$$
\frac{1}{\tau_{\text {eff }}}=\frac{1}{\tau_{s}}+\frac{1}{\tau_{\text {bulk }}},
$$

where $\tau_{\text {bulk }}$ is the bulk lifetime containing Auger, ShockleyRead-Hall, and radiative recombination processes, and $\tau_{s}$ is the surface lifetime representing defect-assisted nonradiative recombination (SRH) at the surfaces of the perovskite film. The observed bulk SRH lifetimes from Sec. III B 1 are derived under the assumption of neglecting surface recombination and, thus, represent the minimum defect-related bulk lifetimes. In the case of non-negligible surface-related recombination, these bulk SRH lifetimes might appear even longer. From the experiment, we are not able to distinguish whether the SRH recombination centers are mainly located in the bulk or at the surfaces. In order to refer to bulk properties instead of those at the surface when performing TRPL measurements, we anneal the perovskite film in ambient air with the aim to passivate potential surface defects. The annealing procedure in ambient air might also (positively) affect the bulk properties in terms of crystal growth and morphology. Because of the typically higher defect densities at the surfaces due to a lack of binding partners, we assume that the surfaces benefit to a greater extend from any passivation effect than the bulk. Additionally, while the surface stays in permanent contact with the ambient atmosphere during the perovskite formation, only a small amount of adsorbed gaseous components might actually enter the bulk by diffusion and, thus, will cause only a minor defect-passivation effect there.

Note that experimentally, the two sample surfaces are made up of a perovskite-glass interface towards the substrate and an exposed perovskite/ $\mathrm{N}_{2}$ interface because the sample is kept in nitrogen during the TRPL measurement. The surface lifetime $\tau_{s}$ is well approximated in the case of an electrical-field-free bulk and equal recombination velocities $S$ at both surfaces by the relation [48]

$$
\tau_{s} \cong \frac{d}{2 S}+\frac{1}{D}\left(\frac{d}{\pi}\right)^{2}
$$

The surface lifetimes according to Eq. (18) are plotted in Fig. 4 as a function of the layer thickness for equal surface recombination velocities at both surfaces. In the case that the bulk will actually be defect-free and, thus, trap-assisted recombination will exclusively occur at surfaces, the surface lifetime $\tau_{s}$ corresponds to the value of the derived SRH bulk lifetime from Sec. III B 3, which is taken here for onesun illumination $\left(\tau_{\mathrm{SRH}} \approx 950 \mathrm{~ns}\right)$ and indicated as a lower limit in Fig. 4. The situation of equal recombination velocities at both surfaces might not exactly hold true because the interfaces of the perovskite layer can exhibit different amounts of interface defects due to different 


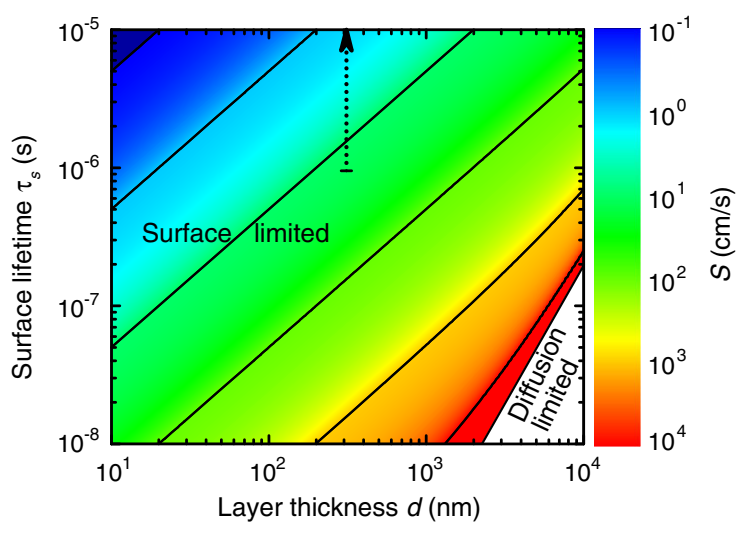

FIG. 4. Surface lifetime $\tau_{s}$ as a function of the layer thickness $d$ for a given mobility of $20 \mathrm{~cm}^{2} \mathrm{~V}^{-1} \mathrm{~s}^{-1}$ and equal surface recombination velocities $S$ at the two surfaces of the sample. The blank area in the lower right corner cannot be occupied even by extreme high surface recombination velocities because chargecarrier diffusion towards the surfaces becomes the limiting process in this region and, thus, is defining the boundary. The dotted black line indicates the possible surface lifetimes for the observed layer thickness of $d=311 \mathrm{~nm}$. The marked origin reflects the lower limit in the case of no present defect-related recombination in the bulk, when $\tau_{s}$ is simply given by the obtained SRH lifetime $\tau_{\mathrm{SRH}} \approx 950 \mathrm{~ns}$ upon one-sun illumination.

adjacent media at each side (glass substrate and nitrogen). In another extreme case, where one of the surface recombination velocities becomes zero due to perfect passivation of defects at one side, $\tau_{s}$ becomes [48]

$$
\tau_{s} \cong \frac{d}{S}+\frac{4}{D}\left(\frac{d}{\pi}\right)^{2}
$$

instead, where $S$ now represents the nonzero surface recombination velocity on the side of the film that is not perfectly passivated. The respective plot according to Eq. (19) is shown in the Supplemental Material [41]. Because of the high derived value of the diffusion constant of the perovskite material according to Eq. (13), the diffusion-related terms in Eqs. (18) and (19) show their impact only for thick layers and single crystals with $d \gg 1 \mu \mathrm{m}$ and when surface recombination velocities are sufficiently high. Interestingly, even if we completely ignore the SRH contribution from the bulk in Eq. (17), already very low surface recombination velocities of some $10-100 \mathrm{~cm} / \mathrm{s}$ are determined for typical observed lifetimes of some hundreds of nanoseconds and typical absorber layer thicknesses. Similar values have been reported for passivated $\mathrm{Si}$ wafers $[49,50]$. A surface recombination velocity of $3400 \mathrm{~cm} / \mathrm{s}$ is obtained for unpassivated perovskite single-crystal surfaces without post-treatment [19], which emphasizes the need for effective perovskite surface passivation, for example, by controlled exposure to oxygen, moisture, chemicals like pyridine [13], or remains of lead iodide [51]. From another perspective, good self-passivation of perovskite surfaces must generally have been achieved for cases when high lifetimes were observed, resulting in remarkably low defect densities in the bulk and, inevitably, also at the surfaces.

\section{Absorption coefficient and intrinsic charge-carrier concentration}

In the following, the radiative recombination parameters are used to determine the corresponding intrinsic chargecarrier concentrations $n_{i}$. We apply the van RoosbroeckShockley relation [52]

$$
k n_{i}^{2}=\int_{0}^{\infty} 4 \pi n_{r}^{2}(E) \alpha(E) \phi_{\mathrm{BB}}(E) d E,
$$

which makes use of the principle of detailed balance: the radiative recombination rate at thermal equilibrium matches the generation rate of electron-hole pairs by thermal radiation. Therefore, the refractive index $n_{r}$, the absorption coefficient $\alpha$, as well as the spectral black-body radiation $\phi_{\mathrm{BB}}$ are needed to calculate the generation rate. This approach relies on accurate data of the absorption coefficient, especially below the band-gap energy of the absorber material, where the absorption is principally weak. Here, we use the generalized Planck emission law introduced by Würfel [53] to first derive the absorptance $A(E)$ of the perovskite film from the spectral PL, and from that, the absorption coefficient $\alpha_{\mathrm{PL}}(E)$ according to Refs. [41,54]. The photon flux $\phi_{\mathrm{PL}}(E)$, which is spontaneously emitted from the sample under nonequilibrium conditions, is related to $A(E)$ by [53]

$$
\phi_{\mathrm{PL}}(E) \propto \frac{A(E) E^{2}}{\exp \left[(E-\Delta \mu) /\left(k_{B} T\right)\right]-1},
$$

where $\Delta \mu$ denotes the quasi-Fermi-level splitting upon illumination. For photon energies $E \gg k_{B} T+\Delta \mu$, the Bose-Einstein term can be approximated by a Boltzmann distribution. Now, a term containing $\Delta \mu$ can be separated, which is completely independent of the photon energy $E$. Consequently, the constant term $\exp \left[\Delta \mu /\left(k_{B} T\right)\right]$ determines only the absolute alignment of the photon flux, but $\Delta \mu$ does not affect its relative shape anymore:

$\phi_{\mathrm{PL}}(E) \propto A(E) E^{2} \exp \left[-E /\left(k_{B} T\right)\right] \exp \left[\Delta \mu /\left(k_{B} T\right)\right]$.

The validity of this approach is shown in the Supplemental Material [41].

The measured PL spectrum of the perovskite film is presented in Fig. 5. In the higher-energetic photon range, where the absorptance $A$ is typically constant, Eq. (22) is used to determine the sample temperature under continuous illumination from the falling PL edge $(T \approx 321 \mathrm{~K})$. Because 


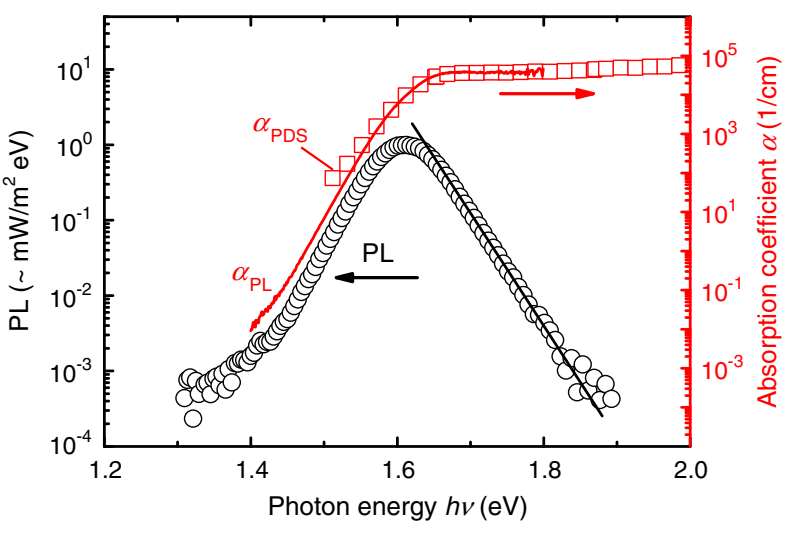

FIG. 5. Photoluminescence spectrum of a $\mathrm{CH}_{3} \mathrm{NH}_{3} \mathrm{PbI}_{3-x} \mathrm{Cl}_{x}$ perovskite thin film (black open circles, left $y$ axis). The black line represents a fit to determine the temperature of the sample. Absorption coefficients (red lines and open squares, right $y$ axis) are obtained by photothermal deflection spectroscopy $\left(\alpha_{\mathrm{PDS}}\right)$ as well as extracted from the photoluminescence spectrum $\left(\alpha_{\mathrm{PL}}\right)$ by applying the generalized Planck radiation law.

the measured PL flux is typically not available in absolute units, the relative values of the absorption coefficient $\alpha_{\mathrm{PL}}$ from the PL shape can simply be adjusted in the high-energetic range to the absolute values obtained by photothermal deflection spectroscopy $\left(\alpha_{\mathrm{PDS}}\right)$. Consequently, precise and ultralow absorption coefficients for perovskite materials over several orders of magnitude can be gained by this approach [55] (see Fig. 5). The observed steep exponential absorption onset indicates a low density of absorption tail states (the so-called Urbach tail). Accordingly, we find a low Urbach energy of $E_{0} \approx 14 \mathrm{meV}$, which corresponds to the slope in the exponential region and is in good agreement with reported values [56]. Based on the obtained absorption coefficients, the intrinsic charge-carrier concentration $n_{i}$ at room temperature $(T=300 \mathrm{~K})$ is obtained according to the van Roosbroeck-Shockley relation [Eq. (20)] and listed in Table II for the cases with and without considering the impact of photon recycling.

\section{Density of states and joint effective mass}

According to the law of mass action [57]

$$
n_{i}^{2}=N_{c} N_{v} e^{-E_{g} /\left(k_{B} T\right)},
$$

values for the product of the density of states in the valence and conduction band $N_{c} N_{v}$ can be provided. Here, a bandgap energy of $E_{g}=1.60 \mathrm{eV}$ is used for the perovskite film. Because the share of each density of states in the product $N_{c} N_{v}$ cannot be separated, we derive a joint effective mass $m_{j}$ instead of an effective mass for each charge-carrier type according to the relation [57]

$$
\sqrt{N_{c} N_{v}}=2\left(\frac{2 \pi m_{j} k_{B} T}{h^{2}}\right)^{3 / 2}
$$

Nevertheless, the determined joint effective mass can be compared to previously reported effective masses because similar values have been reported for electron and hole effective masses [34,40,58]. Consequently, similar density of states $N_{c}$ and $N_{v}$ can be expected. Allowing for photon recycling, our findings (see Table II) with $m_{j}^{*} \approx 0.2$ are in good agreement with values reported in the literature $\left(m_{\mathrm{eff}}^{*} \approx 0.1-0.3\right)[34,40,58]$. When the photon-recycling effect is neglected, we derive a significantly higher mass of $m_{j}^{*} \approx 0.5$, which differs clearly from the literature values.

\section{Prediction of the open-circuit voltage}

Upon illumination at open circuit, all photogenerated charge carriers will eventually recombine. Therefore, the open-circuit voltage $V_{\mathrm{OC}}$ is a suitable parameter which displays recombination losses of all kinds. Shockley and Queisser (SQ) [59] addressed in their approach based on the principle of detailed balance exclusively radiative recombination as the only present recombination loss mechanism. When deriving the open-circuit voltage in the radiative limit according to the SQ theory, only the temperatures of the Sun and the solar cell, as well as the band gap of the absorber material are needed. Here, the absorptance is a step function with full absorption above and zero absorption below the band gap. For the methylammonium lead halide perovskite film, we find a theoretical maximum open-circuit voltage of $V_{\mathrm{OC}}^{\mathrm{rad}}=$ $(1.32 \pm 0.01) \mathrm{V}$ when using absorptance values of a perovskite layer in a hypothetical solar-cell stack (see Sec. III B 3) calculated by ASA. As an extension to the SQ theory, additional nonradiative recombination processes can be taken into account [60-62]. By relating the

TABLE II. Comparison of fundamental semiconductor properties without and with taking photon recycling (PR) into account. The intrinsic charge-carrier concentration $n_{i}$, the product of density of states in the valence and conduction bands $N_{c} N_{v}$, and the joint charge-carrier mass $m_{j}^{*}$ in terms of the electron rest mass are derived by means of the van Roosbroeck-Shockley relation at room temperature $(T=300 \mathrm{~K})$. Additionally, a prediction of the opencircuit voltage $V_{\mathrm{OC}}$ upon one-sun illumination is provided.

\begin{tabular}{lcccc}
\hline \hline & $n_{i}\left(\mathrm{~cm}^{-3}\right)$ & $N_{c} N_{v}\left(\mathrm{~cm}^{-6}\right)$ & $m_{j}^{*}$ & $V_{\mathrm{OC}}(\mathrm{V})$ \\
\hline Without PR & $(3.46 \pm 0.17) \times 10^{5}$ & $(9.1 \pm 5.6) \times 10^{37}$ & $0.524 \pm 0.096$ & $1.24 \pm 0.02$ \\
With PR & $(8.08 \pm 0.39) \times 10^{4}$ & $(4.9 \pm 3.0) \times 10^{36}$ & $0.199 \pm 0.035$ & $1.28 \pm 0.02$ \\
\hline \hline
\end{tabular}


operation of a solar cell to that of a light-emitting device (LED), the attainable open-circuit voltage becomes [62]

$$
V_{\mathrm{OC}}=V_{\mathrm{OC}}^{\mathrm{rad}}+\frac{k_{B} T}{q} \ln \left(Q_{e}^{\mathrm{LED}}\right) .
$$

Here, nonradiative losses are addressed by introducing the external LED quantum efficiency $Q_{e}^{\mathrm{LED}}$ of the solar cell. Electroluminescence measurements can be used as a tool to access $Q_{e}^{\mathrm{LED}}$ and to quantify defect-related losses in perovskite solar cells [63-65]. The external LED quantum efficiency is linked to the internal luminescence quantum efficiency by $[44,66]$

$$
Q_{e}^{\mathrm{LED}}=\frac{p_{e} Q_{i}^{\mathrm{lum}}}{1-Q_{i}^{\mathrm{lum}}\left[1-p_{e}-p_{a}\right]},
$$

with $p_{a}$ being the probability that an emitted photon is parasitically absorbed by the contact layers in the solar-cell stack. Please note that the term $1-p_{e}-p_{a}$ corresponds to the probability $p_{r}$ of photon reabsorption by the absorber layer.

In the following, we show the prediction of the possible $V_{\mathrm{OC}}$ outcome derived from TRPL measurements, which is a simple way to estimate the quality of any absorbing material and its applicability in photovoltaic devices. Because only an absorber layer is investigated, we fortunately do not have to care about any influence of surrounding transport layers on the perovskite interfaces like in a complete photovoltaic device, for instance, in the form of interface states, charge-carrier quenching, interface dipoles, or band bending. Because of the exceptionally low surface recombination velocities, we suppose that the perovskite surfaces in the film used for TRPL are well passivated and nearly defect-free. Consequently, chargecarrier recombination and especially trap-assisted SRH recombination will exclusively occur in the bulk.

In order to derive the $V_{\mathrm{OC}}$ loss due to nonradiative recombination, we calculate the external LED quantum efficiency as well as the individual probabilities occurring in Eq. (26). Because the probability of parasitic photon absorption $p_{a}$ is not easily available, we simply set $p_{a}=0$ in the following. Hereby, we determine an upper limit for $Q_{e}^{\mathrm{LED}}$ and, consequently, also the attainable open-circuit voltage. When we use simulated absorptance values of the perovskite layer embedded in the chosen cell stack, we find an outcoupling efficiency for internally generated photons of $p_{e} \approx 0.056$ according to Ref. [44]. Ultimately, the external LED quantum efficiency becomes $Q_{e}^{\mathrm{LED}} \approx 0.183$, and, according to Eq. (25), we derive a potential open-circuit voltage of $V_{\mathrm{OC}} \approx 1.28 \mathrm{~V}$. Compared to the radiative limit of $V_{\mathrm{OC}}^{\mathrm{rad}} \approx 1.32 \mathrm{~V}$, only approximately $40 \mathrm{mV}$ will be lost due to nonradiative recombination in the bulk, which is an astonishingly low value for a solution-based technology. Some measured open-circuit voltages of perovskite devices with an absorber band gap of $E_{g}=1.6 \mathrm{eV}$ already approach $1.2 \mathrm{~V}$ and, hence, show a low voltage loss [67], whereas organic solar cells typically exhibit significantly higher voltage losses of 0.34-0.44 V [63]. Furthermore, we show in the Supplemental Material [41] that the equation

$$
V_{\mathrm{OC}}=\frac{k_{B} T}{q} \ln \left(\frac{n p}{n_{i}^{2}}\right)=\frac{E_{g}}{q}-\frac{k_{B} T}{q} \ln \left(\frac{N_{c} N_{v}}{n p}\right)
$$

is equivalent to Eq. (25), and we can also use the values with PR in Table II to derive $V_{\text {OC }}$.

As a last step, we quantify the hypothetical impact of photon recycling on the open-circuit voltage. Let us assume for the moment that we can switch off photon recycling in a gedanken experiment. This is equivalent to setting the dominator in Eq. (26) to 1; i.e., Eq. (26) will turn into $Q_{e}^{\mathrm{LED}} \approx p_{e} Q_{i}^{\mathrm{lum}}$. In this scenario, we find $V_{\mathrm{OC}}^{\mathrm{noPR}} \approx 1.24 \mathrm{~V}$. Thus, approximately $40 \mathrm{mV}$ of the derived open-circuit voltage of $V_{\mathrm{OC}} \approx 1.28 \mathrm{~V}$ can be hypothetically attributed to a voltage gain by recycled photons. This estimate is relevant, for instance, to understand the relevance of including photon recycling in analytical and numerical methods aimed to predict efficiency or open-circuit voltage of perovskite solar cells but also to understand how relevant the suppression of parasitic absorption is in order to maximize $V_{\mathrm{OC}}$ by maximizing the amount of photons that can actually be reabsorbed and, thereby, contribute to the photovoltage $[68,69]$.

\section{CONCLUSION}

In summary, we show that TRPL measurements on metal-halide perovskites can provide information that goes beyond the simple extraction of bulk charge-carrier lifetimes. As a first step for a sensible analysis of TRPL transients, we find that mobilities and doping densities need to be determined separately because the diffusion coefficient (or mobility) and thickness will control the effect the surfaces have on the transients, and doping will affect the SRH recombination rate. We determine upper limits for the surface recombination velocities based on mobilities, TRPL lifetimes, and the sample thickness and obtain remarkably low values of about $10 \mathrm{~cm} / \mathrm{s}$. In addition, we observe that TRPL measurements at different laser intensities allow us to distinguish information about firstorder recombination processes (SRH in the bulk or at the surface) from higher-order processes such as radiative and Auger recombination. What is striking in this context is that the radiative recombination coefficient when corrected for photon recycling is relatively high, leading to estimated internal LED efficiencies in the range of $80 \%$ at one sun. From steady-state PL and PDS measurements, we determine effective masses and effective density of states, which further serve to estimate the values for the potential opencircuit voltage that devices with the measured material can 
achieve. Here, we find that for absorber materials exhibiting high internal LED quantum efficiencies, we cannot ignore the impact of photon recycling on the $V_{\mathrm{OC}}$ estimate, and, therefore, accurate information about the optical properties of the device is needed. This result shows that in the future, efforts to suppress parasitic absorption might become relevant for further improvement of open-circuit voltages, which is similar to the case of GaAs solar cells [68].

\section{ACKNOWLEDGMENTS}

The authors acknowledge support from the Deutsche Forschungsgemeinschaft (Grants No. KI-1571/2-1 and No. RA-473/7-1) and from the Helmholtz Association via the Helmholtz Energy Alliance Hybrid PV and the Impulse and Networkfund (HNSEI Project SO-075). We thank Irina Kühn for perovskite layer fabrication as well as Markus Hülsbeck and Jan Flohre for assistance with TRPL and PL measurements. Furthermore, we thank Josef Klomfa $\beta$ and Oliver Thimm for PDS measurements and André Hoffmann for providing optical data.

[1] W. Nie, H. Tsai, R. Asadpour, J.-C. Blancon, A. J. Neukirch, G. Gupta, J. J. Crochet, M. Chhowalla, S. Tretiak, M. A. Alam, H.-L. Wang, and A.D. Mohite, High-efficiency solution-processed perovskite solar cells with millimeterscale grains, Science 347, 522 (2015).

[2] N. J. Jeon, J. H. Noh, W. S. Yang, Y. C. Kim, S. Ryu, J. Seo, and S. I. Seok, Compositional engineering of perovskite materials for high-performance solar cells, Nature (London) 517, 476 (2015).

[3] M. A. Green, K. Emery, Y. Hishikawa, W. Warta, and E. D. Dunlop, Solar cell efficiency tables (version 47), Prog. Photovoltaics 24, 3 (2016).

[4] F. Deschler, M. Price, S. Pathak, L. E. Klintberg, D.-D. Jarausch, R. Higler, S. Hüttner, T. Leijtens, S. D. Stranks, H. J. Snaith, M. Atatüre, R. T. Phillips, and R. H. Friend, High photoluminescence efficiency and optically pumped lasing in solution-processed mixed halide perovskite semiconductors, J. Phys. Chem. Lett. 5, 1421 (2014).

[5] Z.-K. Tan, R. S. Moghaddam, M. L. Lai, P. Docampo, R. Higler, F. Deschler, M. Price, A. Sadhanala, L. M. Pazos, D. Credgington, F. Hanusch, T. Bein, H. J. Snaith, and R. H. Friend, Bright light-emitting diodes based on organometal halide perovskite, Nat. Nanotechnol. 9, 687 (2014).

[6] C. M. Sutter-Fella, Y. Li, M. Amani, J. W. Ager, F. M. Toma, E. Yablonovitch, I. D. Sharp, and A. Javey, High photoluminescence quantum yield in band gap tunable bromide containing mixed halide perovskites, Nano Lett. 16, 800 (2016).

[7] G. Li, Z.-K. Tan, D. Di, M. L. Lai, L. Jiang, J. H.-W. Lim, R. H. Friend, and N. C. Greenham, Efficient light-emitting diodes based on nanocrystalline perovskite in a dielectric polymer matrix, Nano Lett. 15, 2640 (2015).

[8] G. Xing, N. Mathews, S. S. Lim, N. Yantara, X. Liu, D. Sabba, M. Grätzel, S. Mhaisalkar, and T. C. Sum,
Low-temperature solution-processed wavelength-tunable perovskites for lasing, Nat. Mater. 13, 476 (2014).

[9] H. Zhu, Y. Fu, F. Meng, X. Wu, Z. Gong, Q. Ding, M. V. Gustafsson, M. T. Trinh, S. Jin, and X.-Y. Zhu, Lead halide perovskite nanowire lasers with low lasing thresholds and high quality factors, Nat. Mater. 14, 636 (2015).

[10] R. K. Ahrenkiel, Measurement of minority-carrier lifetime by time-resolved photoluminescence, Solid State Electron. 35, 239 (1992).

[11] S. D. Stranks, G. E. Eperon, G. Grancini, C. Menelaou, M. J. Alcocer, T. Leijtens, L. M. Herz, A. Petrozza, and H. J. Snaith, Electron-hole diffusion lengths exceeding 1 micrometer in an organometal trihalide perovskite absorber, Science 342, 341 (2013).

[12] Y. Yamada, T. Nakamura, M. Endo, A. Wakamiya, and Y. Kanemitsu, Photocarrier recombination dynamics in perovskite $\mathrm{CH}_{3} \mathrm{NH}_{3} \mathrm{PbI}_{3}$ for solar cell applications, J. Am. Chem. Soc. 136, 11610 (2014).

[13] D. W. deQuilettes, S. M. Vorpahl, S. D. Stranks, H. Nagaoka, G. E. Eperon, M. E. Ziffer, H. J. Snaith, and D. S. Ginger, Impact of microstructure on local carrier lifetime in perovskite solar cells, Science 348, 683 (2015).

[14] D. Shi, V. Adinolfi, R. Comin, M. Yuan, E. Alarousu, A. Buin, Y. Chen, S. Hoogland, A. Rothenberger, K. Katsiev, Y. Losovyj, X. Zhang, P. A. Dowben, O. F. Mohammed, E. H. Sargent, and O. M. Bakr, Low trap-state density and long carrier diffusion in organolead trihalide perovskite single crystals, Science 347, 519 (2015).

[15] M. Maiberg and R. Scheer, Theoretical study of timeresolved luminescence in semiconductors. I. Decay from the steady state, J. Appl. Phys. 116, 123710 (2014).

[16] M. Maiberg and R. Scheer, Theoretical study of timeresolved luminescence in semiconductors. II. Pulsed excitation, J. Appl. Phys. 116, 123711 (2014).

[17] S. D. Stranks, V. M. Burlakov, T. Leijtens, J. M. Ball, A. Goriely, and H. J. Snaith, Recombination Kinetics in Organic-Inorganic Perovskites: Excitons, Free Charge, and Subgap States, Phys. Rev. Applied 2, 034007 (2014).

[18] E. M. Hutter, G. E. Eperon, S. D. Stranks, and T. J. Savenije, Charge carriers in planar and meso-structured organicinorganic perovskites: Mobilities, lifetimes, and concentrations of trap states, J. Phys. Chem. Lett. 6, 3082 (2015).

[19] Y. Yang, Y. Yan, M. Yang, S. Choi, K. Zhu, J. M. Luther, and M.C. Beard, Low surface recombination velocity in solution-grown $\mathrm{CH}_{3} \mathrm{NH}_{3} \mathrm{PbBr}_{3}$ perovskite single crystal, Nat. Commun. 6, 7961 (2015).

[20] B. Wu, H. T. Nguyen, Z. Ku, G. Han, D. Giovanni, N. Mathews, H. J. Fan, and T. C. Sum, Discerning the surface and bulk recombination kinetics of organic-inorganic halide perovskite single crystals, Adv. Energy Mater. 6, 1600551 (2016).

[21] P. Asbeck, Self-absorption effects on the radiative lifetime in GaAs-GaAlAs double heterostructures, J. Appl. Phys. 48, 820 (1977).

[22] J. You, Y. (M.) Yang, Z. Hong, T.-B. Song, L. Meng, Y. Liu, C. Jiang, H. Zhou, W.-H. Chang, G. Li, and Y. Yang, Moisture assisted perovskite film growth for high performance solar cells, Appl. Phys. Lett. 105, 183902 (2014).

[23] Y. Tian, M. Peter, E. Unger, M. Abdellah, K. Zheng, T. Pullerits, A. Yartsev, V. Sundström, and I. G. Scheblykin, Mechanistic insights into perovskite photoluminescence 
enhancement: Light curing with oxygen can boost yield thousandfold, Phys. Chem. Chem. Phys. 17, 24978 (2015).

[24] R. Ulbricht, E. Hendry, J. Shan, T. F. Heinz, and M. Bonn, Carrier dynamics in semiconductors studied with timeresolved terahertz spectroscopy, Rev. Mod. Phys. 83, 543 (2011).

[25] R. A. Street, Hydrogenated Amorphous Silicon (Cambridge University Press, Cambridge, England, 1991), pp. 224-275.

[26] W. Beyer and H. Overhof, in Semiconductors and Semimetals, edited by J. I. Pankove (Elsevier, New York, 1984), pp. 257-307.

[27] A. C. Boccara, D. Fournier, W. Jackson, and N. M. Amer, Sensitive photothermal deflection technique for measuring absorption in optically thin media, Opt. Lett. 5, 377 (1980).

[28] W. B. Jackson, N. M. Amer, A. C. Boccara, and D. Fournier, Photothermal deflection spectroscopy and detection, Appl. Opt. 20, 1333 (1981).

[29] L. L. Baranowski, K. McLaughlin, P. Zawadzki, S. Lany, A. Norman, H. Hempel, R. Eichberger, T. Unold, E. S. Toberer, and A. Zakutayev, Effects of Disorder on Carrier Transport in $\mathrm{Cu}_{2} \mathrm{SnS}_{3}$, Phys. Rev. Applied 4, 044017 (2015).

[30] G. W. Guglietta, K. R. Choudhury, J. V. Caspar, and J. B. Baxter, Employing time-resolved terahertz spectroscopy to analyze carrier dynamics in thin-film $\mathrm{Cu}_{2} \mathrm{ZnSn}(\mathrm{S}, \mathrm{Se})_{4}$ absorber layers, Appl. Phys. Lett. 104, 253901 (2014).

[31] P. U. Jepsen, W. Schairer, I. H. Libon, U. Lemmer, N. E. Hecker, M. Birkholz, K. Lips, and M. Schall, Ultrafast carrier trapping in microcrystalline silicon observed in optical pump-terahertz probe measurements, Appl. Phys. Lett. 79, 1291 (2001).

[32] R. L. Milot, G. E. Eperon, H. J. Snaith, M. B. Johnston, and L. M. Herz, Temperature-dependent charge-carrier dynamics in $\mathrm{CH}_{3} \mathrm{NH}_{3} \mathrm{PbI}_{3}$ perovskite thin films, Adv. Funct. Mater. 25, 6218 (2015).

[33] C. Wehrenfennig, M. Liu, H. J. Snaith, M. B. Johnston, and L. M. Herz, Charge-carrier dynamics in vapourdeposited films of the organolead halide perovskite $\mathrm{CH}_{3} \mathrm{NH}_{3} \mathrm{PbI}_{3-\mathrm{x}} \mathrm{Cl}_{\mathrm{x}}$, Energy Environ. Sci. 7, 2269 (2014).

[34] G. Giorgi, J.-I. Fujisawa, H. Segawa, and K. Yamashita, Small photocarrier effective masses featuring ambipolar transport in methylammonium lead iodide perovskite: A density functional analysis, J. Phys. Chem. Lett. 4, 4213 (2013).

[35] Q. Wang, Y. Shao, H. Xie, L. Lyu, X. Liu, Y. Gao, and J. Huang, Qualifying composition dependent $p$ and $n$ selfdoping in $\mathrm{CH}_{3} \mathrm{NH}_{3} \mathrm{PbI}_{3}$, Appl. Phys. Lett. 105, 163508 (2014).

[36] C. Wehrenfennig, G. E. Eperon, M. B. Johnston, H. J. Snaith, and L. M. Herz, High charge carrier mobilities and lifetimes in organolead trihalide perovskites, Adv. Mater. 26, 1584 (2014).

[37] Q. Lin, A. Armin, R. C. R. Nagiri, P. L. Burn, and P. Meredith, Electro-optics of perovskite solar cells, Nat. Photonics 9, 106 (2014).

[38] Y. Yang, D. P. Ostrowski, R. M. France, K. Zhu, J. van de Lagemaat, J. M. Luther, and M. C. Beard, Observation of a hot-phonon bottleneck in lead-iodide perovskites, Nat. Photonics 10, 53 (2015).

[39] A. Miyata, A. Mitioglu, P. Plochocka, O. Portugall, J. T.-W. Wang, S. D. Stranks, H. J. Snaith, and R. J. Nicholas, Direct measurement of the exciton binding energy and effective masses for charge carriers in organic-inorganic tri-halide perovskites, Nat. Phys. 11, 582 (2015).

[40] K. Galkowski, A. Mitioglu, A. Miyata, P. Plochocka, O. Portugall, G. E. Eperon, J. T.-W. Wang, T. Stergiopoulos, S. D. Stranks, H. J. Snaith, and R. J. Nicholas, Determination of the exciton binding energy and effective masses for methylammonium and formamidinium lead tri-halide perovskite semiconductors, Energy Environ. Sci. 9, 962 (2016).

[41] See Supplemental Material at http://link.aps.org/ supplemental/10.1103/PhysRevApplied.6.044017 for (i) the long-term PL decay of a doped absorber layer, (ii) optical data of the materials, (iii) a plot of the surface lifetime in the case of $S_{1}=0$ and $S_{2}=S$, (iv) calculation of the absorption coefficient from the absorptance, (v) the validity of the Boltzmann approximation for the generalized Planck emission law, and (vi) a discussion of the validity of Eq. (27).

[42] E. Edri, S. Kirmayer, S. Mukhopadhyay, K. Gartsman, G. Hodes, and D. Cahen, Elucidating the charge carrier separation and working mechanism of $\mathrm{CH}_{3} \mathrm{NH}_{3} \mathrm{PbI}_{3-\mathrm{x}} \mathrm{Cl}_{\mathrm{x}}$ perovskite solar cells, Nat. Commun. 5, 3461 (2014).

[43] Y. Yang, M. Yang, Z. Li, R. Crisp, K. Zhu, and M. C. Beard, Comparison of recombination dynamics in $\mathrm{CH}_{3} \mathrm{NH}_{3} \mathrm{PbBr}_{3}$ and $\mathrm{CH}_{3} \mathrm{NH}_{3} \mathrm{PbI}_{3}$ perovskite films: Influence of exciton binding energy, J. Phys. Chem. Lett. 6, 4688 (2015).

[44] U. Rau, U. W. Paetzold, and T. Kirchartz, Thermodynamics of light management in photovoltaic devices, Phys. Rev. B 90, 035211 (2014).

[45] B. E. Pieters, J. Krc, and M. Zeman, in Proceedings of the 4th WCPEC, Waikoloa, Hawaii, 2006 (IEEE, New York, 2006), pp. 1513-1516.

[46] G. S. Kousik, Z. G. Ling, and P. K. Ajmera, Nondestructive technique to measure bulk lifetime and surface recombination velocities at the two surfaces by infrared absorption due to pulsed optical excitation, J. Appl. Phys. 72, 141 (1992).

[47] T. Otaredian, Separate contactless measurement of the bulk lifetime and the surface recombination velocity by the harmonic optical generation of the excess carriers, Solid State Electron. 36, 153 (1993).

[48] A. B. Sproul, Dimensionless solution of the equation describing the effect of surface recombination on carrier decay in semiconductors, J. Appl. Phys. 76, 2851 (1994).

[49] B. Hoex, S. B. S. Heil, E. Langereis, M. C. M. van de Sanden, and W. M. M. Kessels, Ultralow surface recombination of $c$-Si substrates passivated by plasma-assisted atomic layer deposited $\mathrm{Al}_{2} \mathrm{O}_{3}$, Appl. Phys. Lett. 89, 042112 (2006).

[50] G. Agostinelli, A. Delabie, P. Vitanov, Z. Alexieva, H. F. W. Dekkers, S. De Wolf, and G. Beaucarne, Very low surface recombination velocities on $p$-type silicon wafers passivated with a dielectric with fixed negative charge, Sol. Energy Mater. Sol. Cells 90, 3438 (2006).

[51] Q. Chen, H. Zhou, T.-B. Song, S. Luo, Z. Hong, H.-S. Duan, L. Dou, Y. Liu, and Y. Yang, Controllable self-induced passivation of hybrid lead iodide perovskites toward high performance solar cells, Nano Lett. 14, 4158 (2014).

[52] W. Van Roosbroeck and W. Shockley, Photon-radiative recombination of electrons and holes in germanium, Phys. Rev. 94, 1558 (1954). 
[53] P. Würfel, The chemical potential of radiation, J. Phys. C 15, 3967 (1982).

[54] T. Trupke, E. Daub, and P. Würfel, Absorptivity of silicon solar cells obtained from luminescence, Sol. Energy Mater. Sol. Cells 53, 103 (1998).

[55] C. Barugkin, J. Cong, T. Duong, S. Rahman, H. T. Nguyen, D. Macdonald, T. P. White, and K. R. Catchpole, Ultralow absorption coefficient and temperature dependence of radiative recombination of $\mathrm{CH}_{3} \mathrm{NH}_{3} \mathrm{PbI}_{3}$ perovskite from photoluminescence, J. Phys. Chem. Lett. 6, 767 (2015).

[56] S. De Wolf, J. Holovsky, S.-J. Moon, P. Löper, B. Niesen, M. Ledinsky, F.-J. Haug, J.-H. Yum, and C. Ballif, Organometallic halide perovskites: Sharp optical absorption edge and its relation to photovoltaic performance, J. Phys. Chem. Lett. 5, 1035 (2014).

[57] N. W. Ashcroft and N. D. Mermin, Solid State Physics (Brooks-Cole, Belmont, MA, 1976).

[58] F. Brivio, K. T. Butler, A. Walsh, and M. van Schilfgaarde, Relativistic quasiparticle self-consistent electronic structure of hybrid halide perovskite photovoltaic absorbers, Phys. Rev. B 89, 155204 (2014).

[59] W. Shockley and H. J. Queisser, Detailed balance limit of efficiency of $p-n$ junction solar cells, J. Appl. Phys. 32, 510 (1961).

[60] R. T. Ross, Some thermodynamics of photochemical systems, J. Chem. Phys. 46, 4590 (1967).

[61] G. Smestad and H. Ries, Luminescence and current-voltage characteristics of solar cells and optoelectronic devices, Sol. Energy Mater. Sol. Cells 25, 51 (1992).

[62] U. Rau, Reciprocity relation between photovoltaic quantum efficiency and electroluminescent emission of solar cells, Phys. Rev. B 76, 085303 (2007).
[63] J. Yao, T. Kirchartz, M. S. Vezie, M. A. Faist, W. Gong, Z. He, H. Wu, J. Troughton, T. Watson, D. Bryant, and J. Nelson, Quantifying Losses in Open-Circuit Voltage in Solution-Processable Solar Cells, Phys. Rev. Applied 4, 014020 (2015).

[64] W. Tress, N. Marinova, O. Inganäs, M. K. Nazeeruddin, S. M. Zakeeruddin, and M. Grätzel, Predicting the open-circuit voltage of $\mathrm{CH}_{3} \mathrm{NH}_{3} \mathrm{PbI}_{3}$ perovskite solar cells using electroluminescence and photovoltaic quantum efficiency spectra: The role of radiative and nonradiative recombination, Adv. Energy Mater. 5, 1400812 (2015).

[65] K. Tvingstedt, O. Malinkiewicz, A. Baumann, C. Deibel, H. J. Snaith, V. Dyakonov, and H. J. Bolink, Radiative efficiency of lead iodide based perovskite solar cells, Sci. Rep. 4, 6071 (2014).

[66] U. Rau and T. Kirchartz, On the thermodynamics of light trapping in solar cells, Nat. Mater. 13, 103 (2014).

[67] D. Bi, W. Tress, M. I. Dar, P. Gao, J. Luo, C. Renevier, K. Schenk, A. Abate, F. Giordano, J.-P. Correa Baena, J.-D. Decoppet, S. M. Zakeeruddin, M. K. Nazeeruddin, M. Grätzel, and A. Hagfeldt, Efficient luminescent solar cells based on tailored mixed-cation perovskites, Sci. Adv. 2, e1501170 (2016).

[68] O. D. Miller, E. Yablonovitch, and S. R. Kurtz, Strong internal and external luminescence as solar cells approach the Shockley-Queisser limit, IEEE J. Photovoltaics 2, 303 (2012).

[69] T. Kirchartz, F. Staub, and U. Rau, Impact of photon recycling on the open-circuit voltage of metal halide perovskite solar cells, ACS Energy Lett. 1, 731 (2016). 